Nota científica

\title{
Registro actual del jaguar Panthera onca (Carnivora: Felidae) en el Parque Nacional Cumbres de Monterrey, Nuevo León, México
}

\author{
Jaguar Panthera onca (Carnivora: Felidae) records from Cumbres de Monterrey National Park, \\ Nuevo León, Mexico
}

\author{
Rogelio Carrera-Treviño ${ }^{\text {a }}$, Juan J. Cavazos ${ }^{\mathrm{b}}$, Miguel Briones-Salas ${ }^{\mathrm{c}}$ e Iván Lira-Torres ${ }^{\mathrm{a}, \mathrm{d}, *}$ \\ ${ }^{a}$ Laboratorio de Fauna Silvestre, Facultad de Medicina Veterinaria y Zootecnia, Universidad Autónoma de Nuevo León, \\ Campus Ciencias Agropecuarias, Francisco Villa s/n, Col. Ex-Hacienda El Canadá, 66050, Escobedo, Nuevo León, México \\ ${ }^{\mathrm{b}}$ Domicilio particular: Ignacio Altamirano Núm. 208 Col. Centro, 67350, Allende, Nuevo León, México \\ ${ }^{\mathrm{c}}$ Laboratorio de Vertebrados Terrestres, Mastozoología, Centro Interdisciplinario de Investigación para el Desarrollo Integral Regional, \\ Unidad Oaxaca, Instituto Politécnico Nacional, Hornos 1003, Santa Cruz Xoxocotlán, Oaxaca, México \\ ${ }^{\mathrm{d}}$ Instituto de Ecología Aplicada, Universidad Autónoma de Tamaulipas, Av. División del Golfo Núm. 356, 87019, Ciudad Victoria, Tamaulipas, México
}

Recibido el 11 de marzo de 2015; aceptado el 30 de septiembre de 2015

Disponible en Internet el 26 de febrero de 2016

\begin{abstract}
Resumen
Presentamos los primeros registros de jaguar Panthera onca (Carnivora: Felidae) para el Parque Nacional Cumbres de Monterrey, Nuevo León, México. Entre los años 2013 a 2015 se colocaron cámaras trampa en los bosques templados del parque. Se obtuvieron 15 fotografías y 7 vídeos de varios individuos de la especie, que evidencian la presencia de una población dentro del parque nacional y que representa la población residente de jaguares más al norte de su área de distribución, por la vertiente del golfo de México. Por esta razón resulta necesario fortalecer acciones de protección ambiental para asegurar la conservación de esta población como: promover el pago de servicios ambientales, implementar el pago del seguro ganadero, capacitar a comités de vigilancia comunitarios y atender las denuncias sobre conflictos por depredación de ganado doméstico en esta área.

Derechos Reservados @ 2015 Universidad Nacional Autónoma de México, Instituto de Biología. Este es un artículo de acceso abierto distribuido bajo los términos de la Licencia Creative Commons CC BY-NC-ND 4.0.
\end{abstract}

Palabras clave: Bosques templados; Cámaras trampa; Población de jaguar; Sierra Madre Oriental

\section{Abstract}

We present the first records of jaguar (Panthera onca) in Cumbres de Monterrey National Park, Nuevo León, Mexico. We placed remote cameras between 2013 and 2015 in oak forest habitats of the Park and obtained 15 pictures and 7 videos of 4 different individuals of the species. Our results constitute the first evidence of a resident population of jaguars in this protected area and the northernmost population of the species along the Gulf of Mexico. Strenthenging of conservation measures such as environmental services compensation, livestock compensation programs, conflict management and law enforcement are needed to protect this jaguar population.

All Rights Reserved (C) 2015 Universidad Nacional Autónoma de México, Instituto de Biología. This is an open access item distributed under the Creative Commons CC License BY-NC-ND 4.0.

Keywords: Oak forest; Remote cameras; Jaguar population; Sierra Madre Oriental

\footnotetext{
* Autor para correspondencia.

Correo electrónico: ilira_12@ hotmail.com (I. Lira-Torres).
}

La revisión por pares es responsabilidad de la Universidad Nacional Autónoma de México.
El jaguar Panthera onca (Carnivora: Felidae) es el felino más grande del continente americano y el tercero más grande del mundo (Chávez y Ceballos, 2014; Galindo-Leal, 2009). Es de hábitos nocturnos, dependiendo de la región, y principalmente terrestre y solitario (Moreira et al., 2009). Históricamente se distribuía desde Arizona y Nuevo México, en el sur de Estados 
Unidos de América, hasta Argentina (Seymour, 1989). En México su distribución histórica abarcaba las regiones tropicales y subtropicales desde Sonora y Tamaulipas hasta Chiapas y la península de Yucatán, siguiendo las planicies costeras del golfo y del Pacífico (Hall, 1981). Actualmente se estima que ocupa el 46\% de su área de distribución histórica (Rodríguez-Soto et al., 2011; Swank y Teer, 1989). Las poblaciones más numerosas en México se encuentran en el sureste del país: Campeche, Chiapas, Oaxaca, Yucatán y Quintana Roo (Chávez, Ceballos, Medellín y Zarza, 2007), aunque se ha identificado la porción norte de la Sierra Madre Oriental como una región donde es necesario obtener información sobre la presencia del jaguar (Grigione et al., 2009).

Por sus características crípticas es uno de los animales más difíciles de observar. Por otra parte, ante la creciente problemática de pérdida y fragmentación de hábitat, es una especie que se encuentra en peligro de extinción, ya que requiere de grandes extensiones de hábitat natural para sobrevivir (Chávez y Ceballos, 2014). Aunado a esto, la cacería representa otra gran amenaza para la especie, por lo que actualmente se encuentra clasificado en la Lista roja de especies amenazadas de la Unión Internacional para la Conservación de la Naturaleza (Caso et al., 2008) y, además, considerado en México como en peligro de extinción (Semarnat, 2010).

En el programa de acción para la conservación de la especie: jaguar (Panthera onca), documento rector de la dirección de especies prioritarias de la Comisión Nacional de Áreas Naturales Protegidas, el cual describe las metas, estrategias y acciones a implementar para la conservación del jaguar en México; el Estado de Nuevo León es considerado nivel III entre los sitios prioritarios, debido a que se han presentado registros históricos de la especie y/o los hábitats han sido modificados, de tal forma que es muy probable que las poblaciones se encuentren en grave riesgo de desaparecer y/o sus poblaciones no son muy numerosas (Ceballos et al., 2006; Conanp, 2009), por lo que se recomienda llevar a cabo una evaluación de la situación actual del jaguar en el Estado de Nuevo León para determinar medidas adecuadas para su conservación y manejo (Chávez y Ceballos, 2006).

Desafortunadamente el estudio del jaguar en vida silvestre es complicado y oneroso, incluso a niveles tan elementales como conocer su presencia en un sitio determinado (Briones-Salas, Lavariega y Lira-Torres 2012); aun en aquellos sitios en los que continúa presente, la densidad poblacional tiende a disminuir debido a la persecución que deriva de la hostilidad de la gente (Rabinowitz y Nottingham, 1986; Sanderson et al., 2002; Weber y Rabinowitz, 1996), aunado a que la zona es identificada como de alto riesgo por depredación a nivel nacional (Zarco-González, Monroy-Vilchis y Alaníz, 2013). Esto, sumado a su conducta sigilosa, tiene como resultado que su registro sea cada vez menos frecuente (Gittleman, Funk, Macdonald y Wayne, 2001), por lo que para obtener registros confiables de su presencia es necesario el uso de diferentes dispositivos de captura o registro (BareaAzcón, Virgós, Ballesteros-Duperón, Moleón y Chirosa, 2007).

En Nuevo León la presencia del jaguar ha sido señalada desde diciembre de 1909 (archivo municipal; Jiménez, Contreras y Zúñiga, 1994). Jiménez (1966) describe que a 9.6 km ENE de Zaragoza, un ranchero mató a un «tigre» en el verano de 1964.
Por otro lado, Moreno (1987) tomó fotografías y midió un macho cazado en 1986 al pie de la sierra en Allende. Las observaciones de López-Soto, Rosas-Rosas y Niño-Ramírez (1997); RosasRosas (1996) y Rosas-Rosas y López-Soto (2002) dejan claro que esta especie se distribuyó en el Estado. Existen predicciones que ubican a la especie en el área de estudio (Rodríguez-Soto et al., 2011), así también hay algunos informes anecdóticos que mencionan su presencia en esta área natural, pero creemos que es de la mayor relevancia presentar los primeros registros de $P$. onca en el Parque Nacional Cumbres de Monterrey en el estado de Nuevo León, México, sobre todo porque fortalece la hipótesis propuesta por Grigione et al. (2009), que mencionan que algunas regiones de la Sierra Madre Oriental funcionan como corredores biológicos, y permite el intercambio genético con las poblaciones de jaguares localizadas en el estado vecino de Tamaulipas.

Entre los años 2013 al 2015 se colocaron 7 cámaras trampa marca Bushnell ${ }^{\circledR}$ modelo Trophy Cam de 8 megapíxeles, una cámara trampa marca Moultrie ${ }^{\circledR}$ modelo M-990i de 10 megapíxeles y 4 cámaras trampa marca Wildgame Innovations ${ }^{\circledR}$, modelo M10 de 10 megapíxeles en los parajes conocidos como La Yerbabuena, Los Sabinos y La Ventura en el límite del municipio de Allende y Montemorelos, en el estado de Nuevo León ( $25^{\circ} 11^{\prime} 45.4$ ” N, 100 00'46.0”' O y 25¹0'51.3” N, 99 59'56.7’'O), los mismos que forman parte del Parque Nacional Cumbres de Monterrey y donde la vegetación dominante incluye bosques de Quercus sp. y bosques mixtos de pino-encino (Rzedowsky, 1991) (fig. 1). En estos parajes se desarrolla la cacería cinegética responsable de venado cola blanca (Odocoileus virginianus) y del guajolote norteño (Meleagris gallopavo) y se han tenido registros de avistamiento de jaguar por los cazadores locales, así como indicios de depredación de ganado doméstico atribuidos a esta especie por los rancheros del área (fig. 1).

Las cámaras trampa se colocaron en senderos, manantiales, arroyos, cañadas y saladeros con rastros de presencia de mamíferos, a una altura no mayor de 40 o $50 \mathrm{~cm}$ del nivel del suelo, dependiendo de la topografía e inclinación del área. El circuito de estas cámaras fue programado para permanecer activo durante 24 h, así como para activarse después de 1 min y tomar 3 fotografías y vídeos de $10 \mathrm{seg}$. La posición de cada una de ellas fue georreferenciada con un geoposicionador modelo Garmin etrex ${ }^{\circledR}$. Posteriormente, se revisaron al mes y en cada fotografía detectada se imprimió la hora y fecha. Complementariamente se utilizó como atrayente orina y excreta de jaguar, obtenidas de un criadero particular. El esfuerzo total de muestreo fue de 240 días-trampa.

Se obtuvieron 15 fotografías y 7 vídeos de jaguares, los cuales fueron identificados basándose en su patrón de manchas, que es único para cada individuo (Karanth y Nichols, 1998); esto permitió identificar 4 diferentes ejemplares: un macho (A), 2 hembras (B y C) y un individuo que no pudo ser sexado (D). Las fotografías fueron depositadas en la Colección Nacional de Mamíferos del Instituto de Biología de la UNAM, con el número de catálogo IREKANI-IB-CNM-UNAM-11762, IREKANI-IBCNM-UNAM-11763 y IREKANI-IB-CNM-UNAM-11764. En el caso del macho (A) se obtuvieron 5 vídeos durante el 2013, un vídeo durante 2014, 3 fotografías en serie consecutiva en 


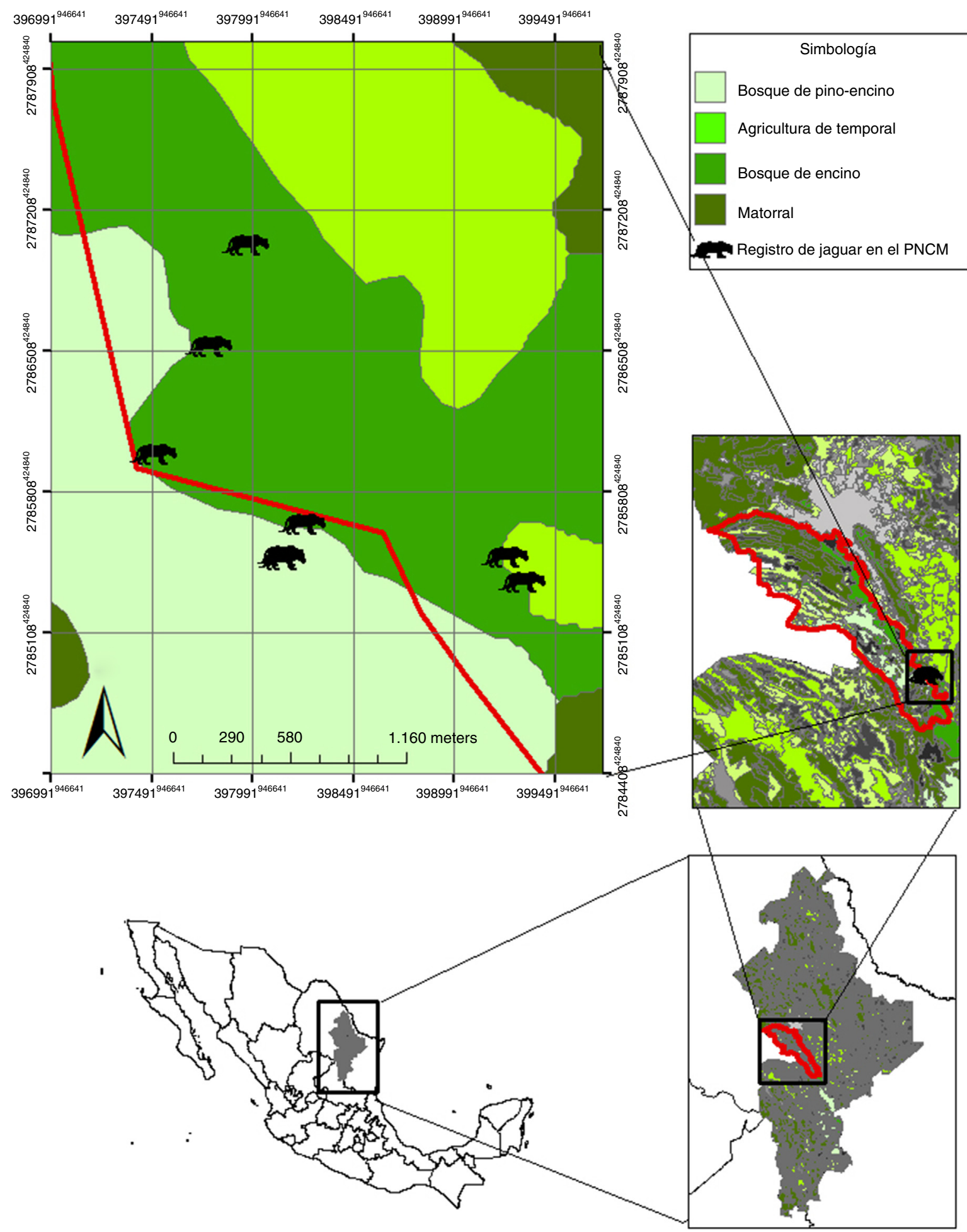

Figura 1. Ubicación de sitios de registro fotográfico del jaguar (Panthera onca) dentro del Parque Nacional Cumbres de Monterrey y su área de influencia en el Estado de Nuevo León, México.

2014 y 3 fotografías en serie consecutiva en 2015. El patrón de actividad registrado para este jaguar es de 20:00 a 05:30 h. En el caso de la primera hembra (B) se obtuvieron 3 fotografías en serie consecutiva durante 2014, a las 15:30 h. Para la segunda hembra (C) se obtuvieron 6 fotografías en serie consecutivas durante 2013, registradas a las 17:00 h. Finalmente, en el caso del individuo no sexado (D), se tiene un video del 30 de junio del 2013, con un registro de actividad a las 05:30 h (fig. 2).

Los parajes donde se obtuvieron los registros se ubican a $10.37 \mathrm{~km}$ al suroeste de la cabecera del municipio de Allende, y a 

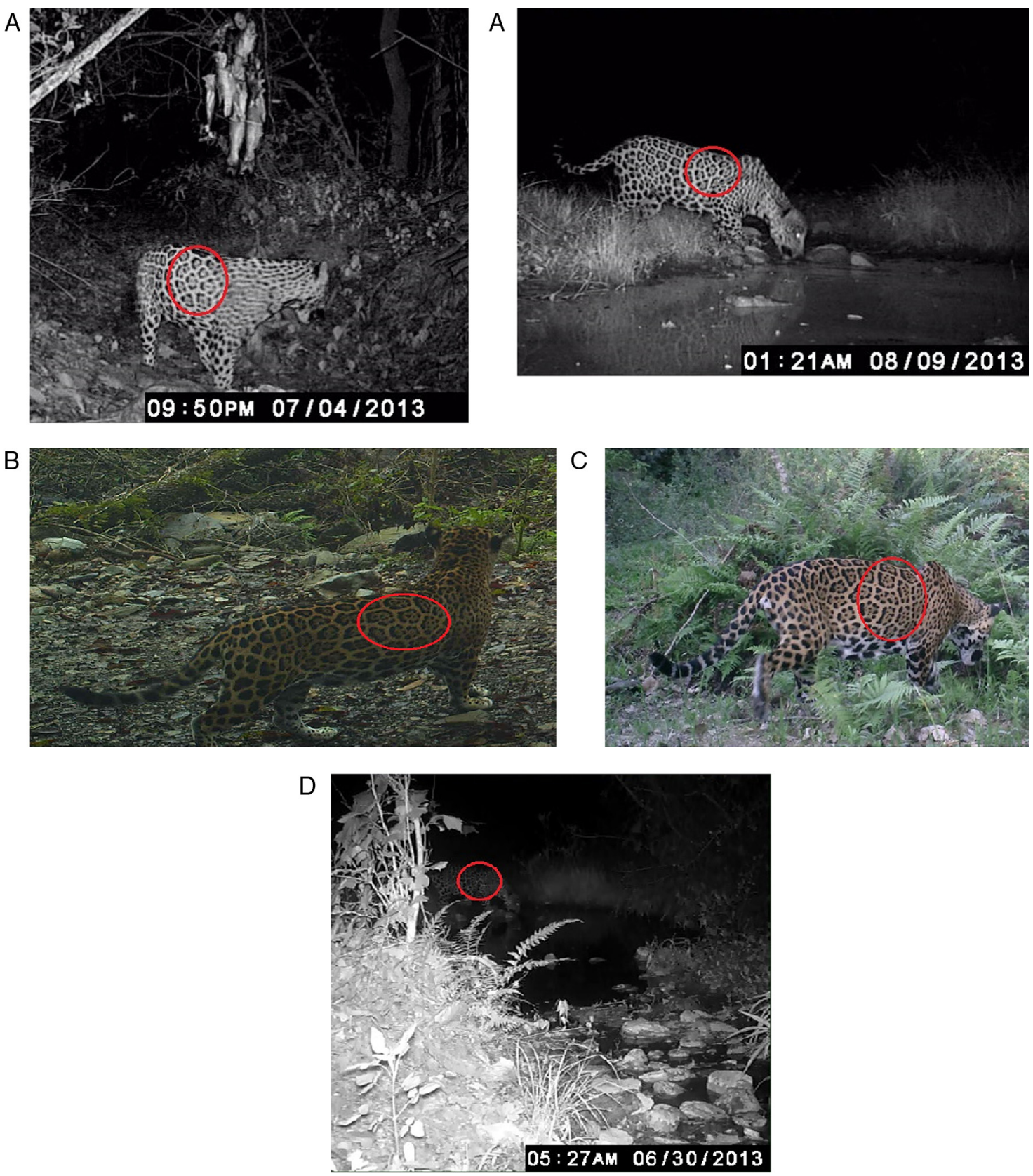

Figura 2. Fotografías para la identificación de individuos de jaguares (Panthera onca) en el Parque Nacional Cumbres de Monterrey, Nuevo León, México. A, jaguar macho adulto; B, jaguar hembra adulta; $\mathrm{C}$, otro individuo de jaguar hembra adulta; D, jaguar adulto de sexo indeterminado.

$62.5 \mathrm{~km}$ al sur del área metropolitana de Monterrey, Nuevo León, y se encuentran en el sureste del polígono del Parque Nacional Cumbres de Monterrey y su zona de influencia. Basándonos en estudios publicados sobre las necesidades biológicas y ecológicas del jaguar (Briones-Salas et al., 2012; Chávez y Ceballos, 2014; López-Soto et al., 1997; Rabinowitz y Nottingham, 1986), sugerimos que la zona sur del Parque Nacional Cumbres de Monterrey cuenta con el hábitat necesario para albergar una población residente y la población más al norte de su área de distribución por la vertiente del golfo de México (Galindo-Leal, 2009). Además, esta región presenta tipos de vegetación templados como Quercus sp. y bosques mixtos de pino-encino, que ya han sido mencionados por otros autores y que funcionan como refugio (Monroy-Vilchis, Sánchez, Aguilera-Reyes, Suárez y Urios, 2008). Asimismo, el área cuenta con la presencia de cuerpos de agua y presas potenciales, que también fueron registradas durante los periodos de muestreo; tlacuache común (Didelphis virginiana), coatí (Nasua narica), mapache (Procyon lotor), venado de cola blanca (O. virginianus) y guajolote norteño (M. gallopavo).

Partiendo de las coordenadas de donde se obtuvieron los presentes registros de jaguar, y la ubicación de los registros históricos en el estado, los mismos se encuentran a $53.6 \mathrm{~km} \mathrm{SSE}$ hacia el municipio de Iturbide, $122 \mathrm{~km}$ SSE hacia el municipio 
de Aramberri y $137 \mathrm{~km}$ SSE hacia el municipio de Zaragoza (López-Soto et al., 1997; Rosas-Rosas, 1996; Rosas-Rosas y López-Soto, 2002), es evidente el vacío de información entre la población de jaguares localizada en el sur del Parque Nacional Cumbres de Monterrey y las poblaciones de jaguar en el estado de Tamaulipas, por lo que es imprescindible continuar las monitorizaciones entre estas 2 regiones y ver el papel de la Sierra Madre Oriental en la conectividad y conservación de esta población a largo plazo (Dueñas-López, 2013).

Finalmente, resulta necesario fortalecer acciones de protección ambiental para asegurar la conservación de esta especie prioritaria, tales como: promover el pago de servicios ambientales, implementar el pago del seguro ganadero, capacitar a comités de vigilancia comunitarios y atender las denuncias sobre conflictos por depredación de ganado doméstico en esta área.

Agradecemos el apoyo de la Comisión Nacional de Áreas Naturales Protegidas (Conanp) a través del Proyecto 4.3: «Prevención y atención al conflicto ganadero con el jaguar (Panthera onca) en el Parque Nacional Cumbres de Monterrey y su zona de influencia», así como a Edgar A. Méndez-Torres y al criadero Manimals por habernos proporcionado las excretas y orina para el presente trabajo. Un reconocimiento muy especial a S. Mireles-Infante y R. Suárez-Hernández por el apoyo técnico, administrativo y la elaboración del mapa. MB-S agradece el apoyo de COFAA, EDI y SIP del IPN. RC, IL-T y MB-S agradecen al Sistema Nacional de Investigadores (SNI) y al Programa para el Desarrollo Profesional Docente para el tipo Superior (Prodep) por su reconocimiento y apoyo.

\section{Referencias}

Barea-Azcón, J. M., Virgós, E., Ballesteros-Duperón, E., Moleón, M. y Chirosa, M. (2007). Surveying carnivores at large spatial scales: a comparison of four broad-applied methods. Biodiversity and Conservation, 16, 1213-1230.

Briones-Salas, M., Lavariega, M. y Lira-Torres, I. (2012). Distribución actual y potencial del Jaguar (Panthera onca) en Oaxaca, México. Revista Mexicana de Biodiversidad, 83, 246-257.

Caso, A., López-González, C., Payan, E., Eizirik, E., de Oliveira, T., Leite-Pitman, R. et al. (2008). Panthera onca. The IUCN Red list of threatened species. Version 2014.2 [consultado 20 Jan 2015]. Disponible en: $\mathrm{http//iucnredlist.org.}$

Ceballos, G., Chávez, C., Blanco, S., Jiménez, R., López, M., Moctezuma, O., et al. (2006). Áreas prioritarias para la conservación. En C. Chávez y G. Ceballos (Eds.), Memorias del primer simposio. El jaguar mexicano en el siglo XXI: situación actual y manejo (pp. 13-19). México D.F.: ConabioAlianza WWF/Telcel-Universidad Nacional Autónoma de México.

Conanp (Comisión Nacional de Áreas Naturales Protegidas). (2009). Programa de acción para la conservación de la especie: jaguar (Panthera onca). México, D.F.: Gobierno Federal y Secretaría de Medio Ambiente y Recursos Naturales.

Chávez, C. y Ceballos, G. (2006). El jaguar mexicano en el siglo XxI: situación actual y manejo. México D.F.: Conabio-Alianza WWF/Telcel-Universidad Nacional Autónoma de México.

Chávez, C. y Ceballos, G. (2014). Jaguar (Panthera onca Linnaeus, 1758). En G. Ceballos (Ed.), Mammals of Mexico (pp. 507-509). Baltimore: Johns Hopkins University Press.

Chávez, C., Ceballos, G., Medellín, R. y Zarza, H. (2007). Primer censo nacional del jaguar. En G. Ceballos, C. Chávez, R. List y H. Zarza (Eds.), Conservación y manejo del jaguar en México: estudios de caso y perspectivas (pp. 113-141). México, D.F.: Conabio-Alianza WWF/Telcel-Universidad Nacional Autónoma de México.

Dueñas-López, G. (2013). Identificación de corredores biológicos potenciales para el jaguar (Panthera onca) en la sierra Abra Tanchipa, San Luis Potosí y sus límites estatales. Tesis de maestría. El Colegio de Postgraduados. San Luis Potosí, México.

Galindo-Leal, C. (2009). Panthera onca. México, D.F: Universidad Autónoma Metropolitana.

Gittleman, J. L., Funk, S. M., Macdonald, D. W. y Wayne, R. K. (2001). Why carnivore conservation? En J. L. Gittleman, S. M. Funk, D. W. Macdonald y R. K. Wayne (Eds.), Carnivore conservation (p. 675). Cambridge: Cambridge University Press.

Grigione, M. M., Menke, K., López-González, C., List, R., Banda, A., Carrera, J., et al. (2009). Identifying potential conservation areas for felids in the USA and Mexico: integrating reliable knowledge across an international border. Fauna y Flora International, Oryx, 43, 78-86.

Hall, R. E. (1981). The mammals of North America. New York: John Wiley y Sons.

Jiménez, G. (1966). Mammals from Nuevo León, Mexico. Tesis de maestría. Kansas: University of Kansas.

Jiménez, G., Contreras, S. y Zúñiga, M. A. (1994). Historia de la mastozoología en Nuevo León, México y su bibliografía. Publicaciones Biológicas, Facultad de Ciencias Biológicas, Universidad Autónoma de Nuevo León, 2, $1-39$.

López-Soto, J. H., Rosas-Rosas, O. y Niño-Ramírez, J. A. (1997). El jaguar (Panthera onca veraecrucis) en Nuevo León México. Revista Mexicana de Mastozoología, 2, 126-128.

Karanth, K. U. y Nichols, J. D. (1998). Estimation of tiger densities in India using photographic captures and recaptures. Ecology, 79, 2852-2862.

Monroy-Vilchis, O., Sánchez, O., Aguilera-Reyes, U., Suárez, P. y Urios, V. (2008). Jaguar (Panthera onca) in the State of Mexico. The Southwestern Naturalist, 53, 533-537.

Moreno, A. (1987). Determinación y distribución de los mamíferos nativos del cañón del Huajuco, Santiago, Nuevo León, México. Tesis. Nuevo León: Facultad de Ciencias Biológicas, Universidad Autónoma de Nuevo León.

Moreira, J., García, R., McNab, R., Ponce-Santizo, G., Mérida, M. y Ruano, G. (2009). Abundancia de jaguares y evaluación de presas asociadas al fototrampeo en las concesiones comunitarias del bloque de Melchor de Mencos. Reserva de la Biosfera Maya, Petén, Guatemala. Guatemala: Informe Final. Wildlife Conservation Society, Programa para Guatemala.

Rabinowitz, A. R. y Nottingham, B. G. (1986). Ecology and behavior of the jaguar (Panthera onca) in Belize, Central America. Journal of Zoology, 210, $149-159$

Rzedowsky, J. (1991). Vegetación de México. México, D.F: Editorial Limusa.

Rodríguez-Soto, C., Monroy-Vilchis, O., Maiorano, L., Boitani, L., Faller, J. C., Briones-Salas, M., et al. (2011). Predicting potential distribution of the jaguar (Panthera onca) in Mexico: identification of priority areas for conservation. Diversity and Distributions, 17, 350-361.

Rosas-Rosas, O. C. (1996). Distribución y aspectos ecológicos del jaguar Panthera onca veraecrusis (Nelson y Goldman 1933) en Nuevo León, México. 1996. Tesis. Nuevo León: Universidad Autónoma de Nuevo León.

Rosas-Rosas, O. y López-Soto, J. H. (2002). Distribución y estado de conservación del jaguar en Nuevo León, México. En R. A. Medellín, C. Equihua, C. Chetkiewics, A. Rabinowitz, P. Crawshaw y K. Redford, et al. (Eds.), El jaguar en el nuevo milenio (pp. 393-401). México, D.F: Fondo de Cultura Económica. Universidad Nacional Autónoma de México y Wildlife Conservation Society.

Sanderson, J., Chetkiewicz, C. L. B., Medellín, R., Rabinowitz, A., Redford, K. H., Robinson, J. G., et al. (2002). Prioridades geográficas para la conservación del jaguar. En R. A. Medellín, C. Equihua, C. L. B. Chetkiewicz, P. G. Crawshaw Jr., A. Rabinowitz y K. H. Redford, et al. (Eds.), El jaguar en el nuevo milenio (pp. 601-628). México, D.F: Fondo de Cultura Económica. Universidad Nacional Autónoma de México y Wildlife Conservation Society.

Semarnat (Secretaría de Medio Ambiente y Recursos Naturales). (2010). Norma Oficial Mexicana NOM-059-ECOL-2010, protección ambiental-especies 
nativas de México de flora y fauna silvestres-Categorías de riesgo y especificaciones para su inclusión, exclusión o cambio-Lista de especies en riesgo. Diario Oficial de la Federación. Jueves 30 de diciembre de 2010, México. Seymour, K. L. (1989). Panthera onca. Mammalian Species, 340, 1-9.

Swank, W. G. y Teer, J. G. (1989). Status of the jaguar. Oryx, 23, 14-21.
Weber, W. y Rabinowitz, A. (1996). A global perspective on large carnivore conservation. Conservation Biology, 10, 1046-1054.

Zarco-González, M. M., Monroy-Vilchis, O. y Alaníz, J. (2013). Spatial model of livestock predation by jaguar and puma in Mexico: conservation planning. Biological Conservation, 159, 80-87. 